\title{
VERSIK PROCESSES AND VERY WEAK BERNOULLI PROCESSES WITH SUMMABLE RATES ARE INDEPENDENT
}

\author{
HEROLD DEHLING, MANFRED DENKER AND WALTER PHILIPP
}

\begin{abstract}
We show that very weak Bernoulli processes and Versik processes having dependence rates $o(1 / n)$ are independent or 2-dependent respectively. The borderline case where the rate is $O(1 / n)$ is discussed in some detail.
\end{abstract}

1. For two probability measures $p$ and $q$ on a Polish space $(S, \sigma)$ let $\mathscr{P}(p, q)$ denote the set of all probability measures on $S \times S$ with marginals $p$ and $q$. The Wasserstein distance $\rho$ of $p$ and $q$ is defined as

$$
\rho(p, q)=\inf \left\{\int \sigma(x, y) d r(x, y): r \in \mathscr{P}(p, q)\right\} .
$$

For two points $x=\left(x_{1}, \ldots, x_{n}\right), y=\left(y_{1}, \ldots, y_{n}\right) \in S^{n}$ we define

$$
\sigma_{n}(x, y):=n^{-1} \sum_{j \leqslant n} \sigma\left(x_{j}, y_{j}\right) \text {. }
$$

We also need to consider the space $S^{n} \times S^{n}$, identified with $\left(S^{2}\right)^{n}$ by the following correspondence $\left(x_{-n}, \ldots, x_{-1}, x_{1}, \ldots, x_{n}\right) \leftrightarrow\left(\left(x_{-1}, x_{1}\right), \ldots,\left(x_{n}, x_{n}\right)\right)$. On $S^{2}$ we define the distance $\sigma^{\prime}$ by setting $\sigma^{\prime}\left(\left(x_{1}, y_{1}\right),\left(x_{2}, y_{2}\right)\right):=\max \left(\sigma\left(x_{1}, y_{1}\right), \sigma\left(x_{2}, y_{2}\right)\right)$ and according to $(+)$ we define the distance on $S^{n} \times S^{n}$, also denoted by $\sigma_{n}$,

$$
\sigma_{n}(x, y)=n^{-1} \sum_{j \leqslant n} \max \left(\sigma\left(x_{j}, y_{j}\right), \sigma\left(x_{-j}, y_{-j}\right)\right)
$$

where $x=\left(x_{-n}, \ldots, x_{-1}, x_{1}, \ldots, x_{n}\right), y=\left(y_{-n}, \ldots, y_{-1}, y_{1}, \ldots, y_{n}\right)$. Throughout this paper let $\left\{X_{j}, j \in \mathbf{Z}\right\}$ be a stationary $S$-valued process defined on the probability space $(\Omega, Q, \mathbf{P})$.

DEFINITION 1. $\left\{X_{j}, j \in \mathbf{Z}\right\}$ is called a Versik process if

$$
V(n):=\rho\left(P_{n}, Q_{n}\right) \rightarrow 0 \quad(n \rightarrow \infty),
$$

where $P_{n}$ and $Q_{n}$ are probability measures on $S^{n} \times S^{n}$ defined by

$$
P_{n}(A \times B)=\mathbf{P}\left\{\left(X_{-n}, \ldots, X_{-1}\right) \in A,\left(X_{1}, \ldots, X_{n}\right) \in B\right\}
$$

and

$$
Q_{n}(A \times B)=\mathbf{P}\left\{\left(X_{-n}, \ldots, X_{-1}\right) \in A\right\} \mathbf{P}\left\{\left(X_{1}, \ldots, X_{n}\right) \in B\right\} .
$$

For discrete random variables this definition is due to Versik (see Rothstein (1980)).

Received by the editors August 4, 1982.

1980 Mathematics Subject Classification. Primary 60A05, 28 D99.

(c) 1984 American Mathematical Society $0002-9939 / 84 \$ 1.00+\$ .25$ per page 
Next for $a \leqslant b, a, b \in \mathbf{Z}$ let $: \pi_{a}^{b}$ be the $\sigma$-field generated by $X_{a}, \ldots, X_{b}$. For

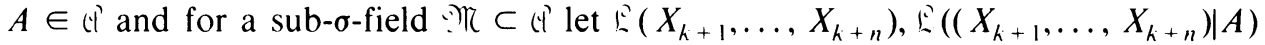
and $:\left(\left(X_{k+1}, \ldots, X_{k+n}\right)\right)$ ) denote respectively the law of $\left(X_{k+1}, \ldots, X_{k+n}\right)$ and the conditional laws of this vector given $A$ or $\rightarrow \pi$.

Definition 2. $\left\{X_{j}, j \in \mathbf{Z}\right\}$ is called very weak Bernoulli (VWB) with rate $\varepsilon(n)$ if $\varepsilon(n) \rightarrow 0$ and if for each $m \geqslant 1$ there exists a set $D=D(m, n) \in \|_{-\infty}^{0}$ with the following properties

(i) $P(D) \geqslant 1-\varepsilon(n)$,

(ii) for all $A \subset D$ with $A \in \eta_{-m}^{()}$we have

$$
\rho\left(\varrho\left(\left(X_{1}, \ldots, X_{n}\right) \mid A\right), \mathcal{L}\left(X_{1}, \ldots, X_{n}\right)\right) \leqslant \varepsilon(n) .
$$

This definition, due to Eberlein (1983) (see also Definition 3 in Gray, Neuhoff and Shields (1975)) coincides with the definition of VWB for discrete random variables $X_{j}$, given by Ornstein $(1974$, p. 18). However, the definition of Ornstein permits a slightly different generalization.

Definition 3. $\left\{X_{j}, j \in \mathbf{Z}\right\}$ is called very weak Bernoulli if

$$
\left.\operatorname{VWB}(n):=\int \rho\left(\mathfrak{L}\left(\left(X_{1}, \ldots, X_{n}\right) \mid \mathfrak{O}\right) \mathbb{R}_{-\infty}^{0}\right), \mathfrak{E}\left(X_{1}, \ldots, X_{n}\right)\right) d \mathbf{P} \rightarrow 0, \quad n \rightarrow \infty .
$$

An application of the Markov inequality shows that if $\left\{X_{j}, j \in \mathbf{Z}\right\}$ is VWB in the sense of Definition 3, then it is also VWB in the sense of Eberlein with rate $\varepsilon(n)=(\operatorname{VWB}(n))^{1 / 2}$. On the other hand if the metric $\sigma$ is bounded both definitions coincide and $\operatorname{VWB}(n) \leqslant \varepsilon(n)(1+\sup \{\sigma(x, y): x, y \in S\})$.

The purpose of this note is to discuss some connections between the rates $V(n), \operatorname{VWB}(n)$ and the rates of decay for certain weak dependence relations commonly used in probability, such as $m$-dependence, absolute regularity and strong mixing in the sense of Rosenblatt.

2. The first and perhaps, at first glance, somewhat surprising result is

THEOREM 1. Let $\left\{X_{j}, j \in \mathbf{Z}\right\}$ be a Versik process with rate $V(n)$. If

$$
\liminf _{n \rightarrow \infty} n V(n)=0
$$

then the process is 2-dependent.

If $\left\{X_{j}, j \in \mathbf{Z}\right\}$ is VWB in the sense of Eberlein with rate $\varepsilon(n)$ satisfying

$$
\liminf _{n \rightarrow \infty} n \varepsilon(n)=0
$$

or if $\left\{X_{j}, j \in \mathbf{Z}\right\}$ is $\mathrm{VWB}$ in the sense of Definition 3 with rate $\mathrm{VWB}(n)$ satisfying

$$
\liminf _{n \rightarrow \infty} n \operatorname{VWB}(n)=0,
$$

then $\left\{X_{j}, j \in \mathbf{Z}\right\}$ is a sequence of independent random variables.

Recall that a process $\left\{X_{j}, j \in \mathbf{Z}\right\}$ is called $m$-dependent if $\mathbf{P}(A \cap B)=\mathbf{P}(A) \mathbf{P}(B)$ for all $A \in \mathfrak{M} \Re_{-\infty}^{a}, B \in \mathfrak{S}_{a+m}^{\infty}$ and $a \in \mathbf{Z}$. We also note that the 2-dependence in the conclusion of the theorem stems from the fact that in the definition of a Versik process $X_{0}$ does not occur. 
The following proof of Theorem 1 is due to Tom Kurtz and is simpler and only half as long as our original proof.

Proof. It follows immediately from the definitions that all the sequences $n V(n)$, $n \varepsilon(n)$ and $n \mathrm{VWB}(n)$ are nondecreasing in $n$ (cf. Gray, Neuhoff and Shields (1975), p. 317). Hence the limits inferior are limits and hence

$$
n V(n)=n \varepsilon(n)=n \operatorname{VWB}(n)=0 \text { for all } n \geqslant 1 \text {. }
$$

Hence by the definition of the Wasserstein distance and Definitions 1,2 and 3 we have $P_{n}=Q_{n}$, L $\left.\left(\left(X_{1}, \ldots, X_{n}\right) \mid A\right)=\mathbb{E}\left(X_{1}, \ldots, X_{n}\right)=E\left(\left(X_{1}, \ldots, X_{n}\right):^{0}\right) \mathbb{C}_{-x_{0}}^{0}\right)$ for all $n \geqslant 1$.

As a consequence of the last theorem, for a nonindependent VWB process or a non 2-dependent Versik process, we must have $\lim _{n \rightarrow \infty} n \mathrm{VWB}(n)>0$ or $\lim _{n \rightarrow \infty} n V(n)>0$ respectively. Hence the next cases to consider are the rates satisfying $\sup n \operatorname{VWB}(n)<\infty$, sup $n \varepsilon(n)<\infty$ and $\sup n V(n)<\infty$. First, we note that processes with such rates exist. In fact stationary, nonindependent Markov chains with finite state space satisfy $\varepsilon(n), \operatorname{VWB}(n)=O\left(n^{-1}\right)$. This may be proved using a simple coupling argument as follows: Let $J$ denote the state space and fix $j_{0} \in J$. If $\left(X_{n}\right)_{n \in \mathbf{Z}}$ is a stationary M.C. let $\left(X_{n}^{*}\right)_{n \geqslant 0}$ be a second M.C. with the same transition probabilities, but with $P\left(X_{0}^{*}=j_{0}\right)=1$. Then $\left\{\left(X_{n}, X_{n}^{*}\right), n \geqslant 0\right\}$ is again a M.C. with state space $J \times J$ and is positive recurrent (see Pitman (1974), Proposition 2.5). For $i \in J$ let $T=\inf \left\{n \geqslant 1:\left(X_{n}, X_{n}^{*}\right)=(i, i)\right\}$. Then $P(T<\infty)=1$ and $E T<\infty$. Define

$$
Y_{n}= \begin{cases}X_{n} & \text { if } n \leqslant T \\ X_{n}^{*} & \text { if } n>T\end{cases}
$$

$Y_{n}$ has the same distribution as $X_{n}$ and $\sum_{i=1}^{m}\left|Y_{i}-X_{i}^{*}\right| \leqslant c T$, hence

$$
E \frac{1}{m} \sum_{i=1}^{m}\left|Y_{i}-X_{i}^{*}\right| \leqslant c \frac{1}{m} E T=O\left(m^{-1}\right),
$$

which proves $\varepsilon(n)=O\left(n^{-1}\right)$ and $\operatorname{VWB}(n)=O\left(n^{-1}\right) .\left(X_{n}\right)$ is even strictly VWB in the sense of Eberlein (1983) with rate $O\left(n^{-1}\right)$.

Since $V(n) \leqslant \mathrm{VWB}(n)$ and since a nonindependent Markov chain is not 2-dependent, this construction also yields a Versik process with sup $n V(n)<\infty$.

This raises the question whether there exists a Versik process with $\sup n V(n)<\infty$ which is not VWB. We do not know the answer to this. However, we have the following result. We first recall two definitions. A stationary process $\left\{X_{j}, j \in \mathbf{Z}\right\}$ is absolutely regular or strongly mixing if

$$
\beta(n):=E \sup _{\{}\left\{\left|P\left(A \mid \Re_{-\infty}^{0}\right)-P(A)\right|: A \in \Re_{n}^{x}\right\} \rightarrow 0 \quad(n \rightarrow \infty),
$$

or

$\alpha(n):=\sup \left\{|P(A \cap B)-P(A) P(B)|: A \in: \eta_{-\infty}^{0}, B \in: \Re_{n}^{x}\right\} \rightarrow 0 \quad(n \rightarrow \infty)$, respectively. 
THEOREM 2. A finite state Versik process $\left\{X_{j}, j \in \mathbf{Z}\right\}$ with rate $V(n)$ satisfying sup $n V(n)<\infty$ is strongly mixing. A finite state $\mathrm{VWB}$ process with rate $\operatorname{VWB}(n)$ satisfying $\sup n \mathrm{VWB}(n)<\infty$ is absolutely regular.

Proof. To prove the first assertion we assume that $\lim _{\sup _{n \rightarrow \infty}} \alpha(n)=a>0$. Then there exist integers $n_{k}$ and $m_{k}$ with $n_{k+1}>m_{k}+n_{k}$ and sets $A_{k} \in$ $\because \pi_{-m_{k}-n_{k}}^{-n_{k}}, B_{k} \in \Omega_{n_{n_{k}}}^{n_{k}+m_{k}}$ such that

$$
\left|P\left(A_{k} \cap B_{k}\right)-P\left(A_{k}\right) P\left(B_{k}\right)\right| \geqslant \frac{1}{2} a \quad(k=1,2, \ldots) .
$$

The sets $A_{k}$ and $B_{k}$ are measurable with respect to $\sigma$-fields with nonoverlapping index sets. Using an argument of Rothstein (1980, p. 215) one can conclude that for each positive integer $N$

$$
\begin{aligned}
\frac{a N}{2} & \leqslant \sum_{k \leqslant N}\left|P\left(A_{k} \cap B_{k}\right)-P\left(A_{k}\right) P\left(B_{k}\right)\right| \\
& \leqslant\left(m_{N}+n_{N}\right) V\left(m_{N}+n_{N}\right) \leqslant \sup n V(n)<\infty,
\end{aligned}
$$

a contradiction.

The proof of the second assertion is similar. We also assume that $\lim _{\text {sup }} \rightarrow x \beta(n)$ $=b>0$. Then there exist integers $n_{k}$ and $m_{k}$ with $n_{k+1}>m_{k}+n_{k}$ such that

$$
\lim _{k \rightarrow \infty} E \sup \left\{\left|P\left(A \mid \Re_{-\infty}^{0}\right)-P(A)\right|: A \in \mathfrak{M}_{n_{k}}^{n_{k}+m_{k}}\right\}=b>0 \text {. }
$$

Next for each $\omega$ we choose a set $A_{k}=A_{k}(\omega) \in \mathcal{N}_{n_{k}}^{n_{k}+m_{k}}$ such that

$$
\begin{aligned}
\mid P\left(A_{k} \mid \mathscr{K}_{-\infty}^{0}\right)(\omega) & -P\left(A_{k}\right) \mid \\
& \geqslant \sup \left\{\left|P\left(A \mid \mathscr{N}_{-\infty}^{0}\right)(\omega)-P(A)\right|: A \in \mathfrak{K}_{n_{k}}^{m_{k}+n_{k}}\right\}-\frac{1}{2} b .
\end{aligned}
$$

Then, using the same arguments mentioned above, we obtain for each positive integer $N$

$$
\begin{aligned}
& N\left[\sup \left\{\left|P\left(A \mid \mathfrak{T}_{-\infty}^{0}\right)(\omega)-P(A)\right|: A \in \mathfrak{N}_{n_{k}}^{n_{k}+m_{k}}\right\}-\frac{1}{2} b\right] \\
& \quad \leqslant \sum_{k \leqslant N}\left|P\left(A_{k} \mid \mathfrak{N} \mathbb{R}_{-\infty}^{0}\right)(\omega)-P\left(A_{k}\right)\right| \\
& \quad \leqslant\left(m_{N}=n_{N}\right) \rho\left(\mathcal{L}\left(\left(X_{1}, \ldots, X_{m_{N}+n_{N}}\right) \mid \mathfrak{N}_{-\infty}^{0}\right), \varrho\left(X_{1}, \ldots, X_{m_{N}+n_{N}}\right)\right) .
\end{aligned}
$$

We integrate this inequality with respect to $P(d \omega)$ and obtain the desired contradiction.

REMARK. Theorem 2 does not hold for general state space. In fact, Bradley (1983) recently succeeded in constructing an example of a process with a state space with bounded metric which is very weakly Bernoulli (in both senses of the present note) with rate $O\left(n^{-1}\right)$, which is not even strongly mixing.

The following result is a partial converse to Theorem 2.

THEOREM 3. Let $\boldsymbol{\sigma}$ be a bounded metric. If $\left\{X_{j}, j \in \mathbf{Z}\right\}$ is absolutely regular then it is VWB in the sense of Definition 3. If $\left\{X_{j}, j \in \mathbf{Z}\right\}$ is strongly mixing then it is also Versik. 
Proof. Let $\left\{X_{j}, j \in \mathbf{Z}\right\}$ be absolutely regular. Fix $n$ and $\omega$ and set

$$
\beta(n, \omega):=\sup \left\{\left|P\left(A \mid: \eta_{-\infty}^{0}\right)(\omega)-P(A)\right|: A \in: \eta \chi_{n}^{x}\right\} .
$$

Then by a standard argument we obtain for each bounded, $\because{ }_{n}^{x}$-measurable function $f$

$$
\left|\int f d P\left(\cdot \mid \mathbb{O}_{-\infty}^{0}\right)(\omega)-\int f d P\right| \leqslant 2\|f\|_{\infty} \beta(n, \omega) .
$$

For fixed $m \geqslant 1$ we write

$$
\mu_{\omega}:=L^{\prime}\left(\left(X_{n+1}, \ldots, X_{n+m}\right) \mid \cdots \mathbb{R}_{-x}^{0}\right)(\omega) \text { and } \mu:=\mathbb{L}^{\prime}\left(X_{n+1}, \ldots, X_{n+m}\right) \text {. }
$$

We recall the Lipschitz norm $\|f\|_{I}:=\sup \left\{\mid f(x)-f(y)(\sigma(x, y))^{-1}: x \neq y\right\}$. Then by (*) and the Kantorovich-Rubinstein theorem (see Dudley (1976), Section 20.1) we obtain for the Wasserstein distance of $\mu_{\omega}$ and $\mu$

$$
\begin{aligned}
\rho\left(\mu_{\omega}, \mu\right) & =\sup \left\{\left|\int f d P\left(\cdot \mid \Re_{-\infty}^{0}\right)(\omega)-\int f d P\right|: f \in: \prod_{n}^{m+n},\|f\|_{L} \leqslant 1\right\} \\
& \leqslant 2 \beta(n, \omega) \max \{\sigma(x, y): x, y \in S\}=C \beta(n, \omega),
\end{aligned}
$$

say, for some $C>0$. Let

$$
\nu_{\omega}:=L^{\prime}\left(\left(X_{1}, \ldots, X_{n+m}\right) \mid \because \mathbb{R}_{-x}^{0}\right)(\omega) \text { and } \nu=\mathbb{L}\left(X_{1}, \ldots X_{n}, m\right) \text {. }
$$

For each $\lambda \in \varphi^{\top}\left(\mu_{\omega}, \mu\right)$ we can construct a $\lambda^{*} \in\left(P\left(\nu_{\omega}, \nu\right)\right.$ by using Lemma Al of Berkes and Philipp (1979) according to the following diagram

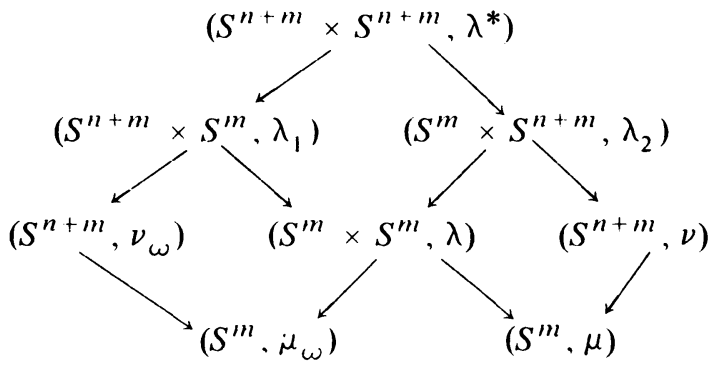

where the arrows stand for the operation of passing to a marginal distribution on an appropriate state space.

Now

$$
\int \frac{1}{m+n} \sum_{1 \leqslant m+n} \sigma\left(x_{i}, y_{j}\right) d \lambda^{*}(x, y) \leqslant C\left(\frac{n}{m+n}+\frac{2 m \beta(n, \omega)}{m+n}\right) .
$$

Integrating with respect to $\omega$ we obtain

$$
\begin{aligned}
& \int \rho\left(\mathbb{E}\left(\left(X_{1}, \ldots, X_{m+n}\right) \mid M_{-x}^{0}\right), \stackrel{E}{ }\left(X_{1}, \ldots, X_{m+n}\right)\right) d \mathbf{P}(\omega) \\
& \leqslant C\left(\frac{n}{m+n}+\frac{2 m \beta(n)}{m+n}\right) \rightarrow 0
\end{aligned}
$$

if $m \sim n \beta^{1 / 2}(n)$ and $n \rightarrow x$. 
The case of Versik processes is proved similarly.

We conclude with a comparison between a VWB process and a Versik process. It is easy to see that every $\mathrm{VWB}$ process is $\operatorname{Versik}$ and $V(n) \leqslant \operatorname{VWB}(n)$. This is suggested by Theorems 2 and 3 (since every absolutely regular process is strongly mixing), and a proof that the above assertion is indeed true can be easily modeled after the proof of Theorem 3. These two theorems also suggest that there are Versik processes which are not VWB, since there are strongly mixing processes which are not absolutely regular. In the discrete case these comparisons between VWB and Versik processes are due to Rothstein (1980), who constructed a zero entropy Versik process with discrete state space which clearly cannot be VWB. Rothstein's (1980) example can be easily extended to continuous state spaces by the following result.

Proposition 4. Let $\left\{X_{j}, j \in \mathbf{Z}\right\}$ be a VWB process or a Versik process and let $\left\{Y_{j}, j \in \mathbf{Z}\right\}$ be a sequence of independent, identically distributed random variables independent of $\left\{X_{j}, j \in \mathbf{Z}\right\}$. Then $\left\{\left(X_{j}, Y_{j}\right), j \in \mathbf{Z}\right\}$ is also a VWB process or a Versik process respectively with the same rates as the original processes. Here the metric on the product space is the maximum metric.

Proof. We shall give the proof for Versik processes. The VWB case is proved similarly.

Denote by $(S, \sigma)$ the state space for $\left\{X_{j}, j \in \mathbf{Z}\right\}$ and by $(\hat{S}, \hat{\sigma})$ that one of $\left\{Y_{j}, j \in \mathbf{Z}\right\}$. Let $P_{n}$ and $Q_{n}$ denote the distributions defined in Definition 1 for the process $\left\{X_{j}, j \in \mathbf{Z}\right\}$, and let $r \in \mathcal{P}\left(Q_{n}, P_{n}\right)$. If $R_{n}$ denotes the distribution of $Y_{-n}, \ldots, Y_{-1}, Y_{1}, \ldots, Y_{n}$, then $r \times \bar{R}_{n}$ has marginals $P_{n} \times R_{n}$ and $Q_{n} \times R_{n}$, where $\bar{R}_{n}$ denotes the distribution $R_{n}$ on the diagonal of $\left(\hat{S}^{n} \times \hat{S}^{n}\right) \times\left(\hat{S}^{n} \times \hat{S}^{n}\right)$. It is also clear that for the Versik coefficients $V^{\prime}(n)$ of $\left\{\left(X_{j}, Y_{j}\right), j \in \mathbf{Z}\right\}$ we have

$$
\begin{aligned}
V^{\prime}(n) \leqslant \int n^{-1} \sum_{j \leqslant n} \max \left\{\sigma\left(x_{j}, y_{j}\right), \sigma\left(x_{-j}, y_{-j}\right),\right. \\
\left.\hat{\sigma}\left(u_{j}, v_{j}\right), \hat{\sigma}\left(u_{-j}, v_{-j}\right)\right\} d r \times \bar{R}_{n}(x, y, u, v) \\
=\int n^{-1} \sum_{j \leqslant n} \max \left\{\sigma\left(x_{j}, y_{j}\right), \sigma\left(x_{-j}, y_{-j}\right)\right\} d r(x, y) .
\end{aligned}
$$

Hence $V^{\prime}(n) \leqslant V(n)$, where $V(n)$ denotes the Versik coefficient of $\left\{X_{j}, j \in \mathbf{Z}\right\}$. $V(n) \leqslant V^{\prime}(n)$ is trivial.

\section{REFERENCES}

I. Berkes and W. Philipp (1979), Approximation theorems for independent and reakly dependent random vectors, Ann. Probab. 7, 29-54.

R. C. Bradley (1983), On a very weak Bernoulli condition, preprint.

R. M. Dudley (1976), Prohabilties and metrics, Aarhus Univ. Math. Inst. Lecture Notes Ser., No. 45. Aarhus.

E. Eberlein (1983), Strong approximation of very weak Bernoulli processes, Z. Wahrsch. Verw. Gebiete 62, 17-37.

R. M. Gray, D. L. Neuhoff and P. C. Shields (1975), A generalisation of Ornstein's $\bar{d}$-distance with applications to information theory, Ann. Probab. 3, 315-328. 
D. Ornstein (1974), Ergodic theory, randomness, and d!namical șstems, Yale Math. Mono.. No. 5. Yale Univ. Press, New Haven.

J. W. Pitman (1974), Uniform rates of convergence for Markor chain transition prohahilities, Z. Wahrsch. Verw. Gebiete 29, 193-227.

A. Rothstein (1980), Versik processes: First steps, Israel J. Math. 36, 205-223.

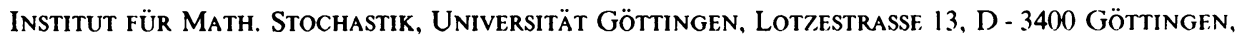
Federal Republic of Germany (Current address of Herold Dehling and Manfred Denker)

Department of Mathematics, University of Illinois, Urbana, Illinois 61801 (Current address of Walter Philipp) 